\title{
3 Research Square

\section{Polydatin Protects Against Articular Cartilage Degeneration by Regulating Autophagy Mediated by AMPK/Mtor Signaling Pathway}

\section{Zhengcong Ye}

Hangzhou Xiaoshan District Hospital of Traditional Chinese Medicine

\section{Chun He}

Hangzhou Xiaoshan District Hospital of Traditional Chinese Medicine

Pengzheng Yu

Hangzhou Xiaoshan District Orthopedics and Traumatology Hospital

\section{Guoping Cao}

Hangzhou Xiaoshan District Hospital of Traditional Chinese Medicine

\section{Qinrong Shen}

Shaoxing Hospital of Traditional Chinese Medicine

\section{Canfeng Wang ( $\triangle$ Wangchanfeng123@163.com )}

Department of orthopedic,Hangzhou Xiaoshan District Hospital of Traditional Chinese Medicine

\section{Research article}

Keywords: knee osteoarthritis, polydatin, autophagy, apoptosis

Posted Date: July 19th, 2021

DOI: https://doi.org/10.21203/rs.3.rs-708886/v1

License: () (1) This work is licensed under a Creative Commons Attribution 4.0 International License.

Read Full License 


\section{Abstract}

Background. Knee osteoarthritis (KOA) is one of the leading causes of disability, and its etiopathogenesis is not completely understood. Polydatin has the potential effect on the treatment of KOA, but the mechanism is not clear.

Methods. After an KOA rat model was established by anterior cruciate ligament transection, KOA rats were treated with polydatin $(4 \mathrm{mg} / \mathrm{kg})$ for 30 days. Subsequently, cartilage tissues were collected from rats and detected by HE, TUNEL staining and Western blotting to evaluate the pathological damage, apoptosis and autophagy activity. Then, human chondrocyte C28/I2 cells were stimulated by LPS to induce a KOA model in vitro, and the effects of polydatin on the C28/I2 cell viability, apoptosis and autophagy were also detected. In addition, the mechanism of polydatin on KOA in C28/I2 cells was investigated, and the effect of an AMPK inhibitor (Dorsomorphin $2 \mathrm{HCl}$ ) on the proliferation and apoptosis of polydatin administrated-cells were also detected.

Results. After treated with polydatin, the pathological damage of rat cartilage tissues were ameliorated, cells apoptosis was inhibited and autophagy was activated in KOA rats. Meanwhile, polydatin also ameliorated the proliferation and apoptosis of $\mathrm{C} 28 / \mathrm{I} 2 \mathrm{cells}$, the expression of autophagy-related proteins, LC3II/LC3I, Beclin-1, and p-AMPK/AMPK were up-regulated, p-mTOR/mTOR was down-regulated by polydatin in $\mathrm{C} 28 / \mathrm{I} 2$ cells. Interestingly, relative results showed that the improvement effect of polydatin on LPS-sdtimulated-C28/I2 cells was blocked by AMPK/mTOR inhibitor, Dorsomorphin $2 \mathrm{HCl}$.

Conclusion: Our research showed that polydatin reduces apoptosis and activate autophagy both in a rat model of KOA and C28/I2 cell model by AMPK/mTOR signaling pathway, which provides the basis for further investigations into the potential therapeutic impact of polydatin in KOA.

\section{Introduction}

Knee osteoarthritis (KOA) is a multifactorial disease characterized by decreased muscle strength, limited function and narrowed joint spaces or even disability caused by severe pain [1]. It is a degenerative joint disease and often related to age, and the prevalence is increased followed the increased of age [2]. As the global population aging, KOA is becoming an increasing burden on society. At present, the therapeutic methods for KOA are limited and the most common treatments were analgesics and physicaltherapy. Unfortunately, joint replacement or osteotomy may be inevitable [3]. Therefore, it is urgent for researchers and practitioners to search a new prevention and treatment of KOA.

Owning to the complexity of KOA pathology, the main causes leading to KOA are still unclear [4]. The key factors of the degeneration of articular cartilage are considered as the extracellular matrix lost and chondrocytes died in KOA [5]. Usually, cells could adapt to the pressures of environmental by regulate autophagy and apoptotic. The apoptosis of extracellular matrix which synthesized and secreted from articular chondrocytes was associated with the pathogenesis of KOA [6]. At the same time, chondrocyte 
autophagy also acts a key role in the process of KOA [7]. Therefore, the treatment which associated with the dysregulating apoptosis and autophagy of chondrocyte is useful.

Polydatin is isolated from the plant Polygonum cuspidatuma, and is a kind of glycosylated polyphenol with a monocrystalline structure. It has a lot of positive health functions such as immunoregulatory, antiinflammatory, anti-oxidative and anti-tumoral proprieties [8, 9]. In addition, polydatin can regulate the autophagy mediated by AMPK/mTOR signaling pathway. It can inhibit the proteins expression of $\mathrm{PI} 3 \mathrm{~K} / \mathrm{Akt} / \mathrm{mTOR}$ pathway to improve autophagic dysfunction and reduce atherosclerotic lesions [10]. And it also suppressed autophagy to protect against fructose-induced podocyte injury by its anti-oxidation activity [11].

Therefore, we established a KOA model both in vivo and in vitro to investigate the possibility effect of polydatin for the treatment of KOA. Then the regulation of polydatin on apoptosis and autophagy was investigated, and the potential mechanism was further explored.

\section{Materials And Methods}

\section{In vivo}

\section{Animals}

Thirty healthy adult Sprague-Dawley (SD) rats (250 280 g) were purchased from the Shanghai SLAC Laboratory Animal Co., Ltd. (SCXK (Hu) 2017-0005, Shanghai, China). All the rats were allowed to access food and water free and living in standard condition (humidity: $55 \sim 70 \%$; room temperature: $23 \pm 2{ }^{\circ} \mathrm{C}$; light cycle: 12-h light/dark). All animal assays were performed according to the National Institutes of Health Guide for the Care and Use of Laboratory Animals. The animal experiments were in accordance with the guidelines of laboratory animal care and were approved by the Hangzhou Eyong Biotechnological Co., Ltd. Animal Experiment Center (Hangzhou, China).

\section{Established KOA model and experimental group}

The rats were divided into 3 groups randomly ( $n=10 /$ group): control, KOA and KOA + polydatin. The KOA was induced according to previously study [12]. In detail, after anesthetized with pentobarbital (40 $\mathrm{mg} / \mathrm{kg}$ ), the KOA and KOA + polydatin rats were fixed in supine position. And the right medial knee joint was made a longitudinal incision $(1.5 \mathrm{~cm})$, the medial collateral ligament and joint capsule was exposed and broken. Then dislocated the patella to expose and cut off the anterior cruciate ligament. Later, the medial meniscus was extirpated and cut off the posterior crucial ligament. Finally, established KOA rat models were verified by drawer test. After fixed the patella, sutured the joint capsule and skin. In order to avoid infection, all the rats were treated with penicillin $(40,000 \mathrm{U} / \mathrm{mL}, 1 \mathrm{~mL})$ by intramuscularly injected after the surgery for consecutive 3 days. The normal group rats not received any treatment. All the rats were fed and drank freely and normally. After modeling 4 weeks [13], the KOA + polydatin rats were 
intraperitoneally injected with polydatin ( $4 \mathrm{mg} / \mathrm{kg}$, dissolved in saline), while normal rats and model rats intraperitoneally injected with same volume normal saline and continued for $30 \mathrm{~d}$.

\section{Hematoxylin-eosin (HE) staining}

The cartilage tissues of each group rats were fixed in $4 \%$ paraformaldehyde for $24 \mathrm{~h}$ at room temperature. Then, the cartilage tissues were routinely dehydrated by gradient ethanol, embedded, and cut into $4 \mu \mathrm{m}$ sections. Then the sections were stained with HE. After washed, the slices were observed under an optical microscope (Olympus, Japan) to detected the histopathological changes. The cartilage tissues were scored as following: 0 point, normal; 1 point, mild cell infiltration and synovial invasion; 2 points, bone invasion; 3 points, more serious cell infiltration and synovial invasion accompanied by bone invasion; 4 points, severe cell infiltration and synovial invasion accompanied by bone invasion.

\section{Terminal deoxynucleotidyl transferase dUTP nick end labeling (TUNEL) assay}

The apoptosis rate of chondrocytes was assessed by TUNEL assay. After fixed with $4 \%$ paraformaldehyde and dehydrated, the brain tissue was embedded and sectioned into 4- $\mu \mathrm{m}$. When the slices were dewaxed and transparent, $100 \mu \mathrm{L} \mathrm{TdT}$ enzyme buffer was added and incubated at $37^{\circ} \mathrm{C}$ for 1 $\mathrm{h}$ in a dark and humectation environment. Then the slices were incubated with $100 \mu \mathrm{L}$ Streptavadin-HRP at $37^{\circ} \mathrm{C}$ for $30 \mathrm{~min}$. After washed, the sections were stained with diaminobenzidine (DAB) (MD912068, MDL, China), and re-stained with hematoxylin. Six fields were randomly selected for each group and quantitative analysis was detected by Image J. Apoptosis index (\%) = TUNEL-positive neurons/total neurons $\times 100 \%$.

\section{Western blotting}

Radioimmunoprecipitation assay (RIPA) was used to extracted protein from the ischemic cartilage tissues. After crushed in liquid nitrogen, the tissue lysates were collected and centrifugated at $12000 \mathrm{~g}$ for 20 min to obtained the supernatant. After quantified the concentration of total cellular proteins by bicinchoninic acid (BCA, pc0020, Solarbio, China) kit. The proteins were loaded onto SDS-PAGE gel, separated by electrophoresis, and transferred onto a polyvinylidene difluoride (PVDF) membrane (GE Healthcare Life, USA). After blocked by $5 \%$ dried skim milk for $30 \mathrm{~min}$, primary antibodies were added and incubated with membranes overnight at $4{ }^{\circ} \mathrm{C}$, then incubated with secondary antibody at room temperature for $1 \mathrm{~h}$. Enhanced chemiluminescence (ECL) (Solarbio, Beijing, China) method was used to detected the expression of protein. Image $\mathrm{J}$ software (National Institutes of Health) was used to detected the band intensities. The relative protein level was normalized to that of GAPDH. The primary antibodies including anti-LC3A/B antibody (1:500, AF5402, Affinity, USA), anti-Beclin 1 antibody (1:500, AF5128, Affinity, USA), anti-GAPDH antibody (1:3000, AF7021, Affinity, USA).

\section{In vitro}

Cell culture and grouping 
C28/I2 cell lines were cultured in DMEM/F12 medium (SH30243.01, Hyclone, USA) at $37^{\circ} \mathrm{C}$ and under $5 \%$ $\mathrm{CO}_{2}$ atmosphere. The medium was changed every $48 \mathrm{~h}$. When the cells reached about $80-90 \%$ confluence, cells were used for the further experiments. The $\mathrm{C} 28 / \mathrm{I} 2$ cell lines were seeded into six plates or flasks and divided into 3 groups: control (only DMEM/F-12), LPS (DMEM/F-12 contain $5 \mu \mathrm{g} / \mathrm{mL}$ LPS, cultured for 24 h), LPS+polydatin (DMEM/F-12 contain $5 \mu \mathrm{g} / \mathrm{mL}$ LPS and $1 \mu \mathrm{M}$ polydatin, cultured for $24 \mathrm{~h}$ ) [14].

\section{MTT}

In order to detected the effect of LPS and polydatin on C28/I2 cells, MTT assay was performed. Briefly, C28/I2 cells $\left(1 \times 10^{5} \mathrm{cell} / \mathrm{L}, 100 \mu \mathrm{l} /\right.$ well) which was logarithmic growth phase were seeded into 96 -well plates and incubated in a incubator $\left(37^{\circ} \mathrm{C}, 5 \% \mathrm{CO}_{2}\right)$ for $24 \mathrm{~h}$. After treated with LPS and polydatin for $24 \mathrm{~h}$, $10 \mu \mathrm{L}$ MTT was added and cultured for another $24 \mathrm{~h}$. Finally, the plates were detected at $450 \mathrm{~nm}$.

\section{Flow Cytometry}

Logarithmic growth phase C28/I2 cells $\left(1.2 \times 10^{6} /\right.$ well) were seeded into 96 -well plates. After induced by LPS and treated with polydatin for $24 \mathrm{~h}$, the concentration of C28/I2 cells were diluted into $1 \times 10^{6}$ cells $/ \mathrm{mL}$ by cool PBS. Then, annexin V/PI staining was processed to assess the anti-apoptotic effects of polydatin on C28/I2 cells induced by LPS. C28/I2 cells were re-suspend in binding buffer $(100 \mu \mathrm{L})$, and co-incubated with Annexin V-FITC $(5 \mu \mathrm{L})$ and PI solution $(10 \mu \mathrm{L})$ at room temperature in the dark for $15 \mathrm{~min}$. After added $400 \mu \mathrm{L}$ binding buffer, the solution was analyzed by flow cytometry within 1 h.

\section{Western blotting}

After treated with LPS and polydatin, the C28/I2 cells were lysed with RIPA buffer to obtained protein. After measured the concentrations by BCA Kit, proteins were also separated by SDS-PAGE and transferred onto PVDF membranes. After blocked with $5 \%$ dried skim milk for $2 \mathrm{~h}$, the membranes were incubated with dilute solution of antibodies at $4{ }^{\circ} \mathrm{C}$ overnight and secondary antibody for $2 \mathrm{~h}$ at room temperature. ECL method was used to detected the expression of protein. Image $\mathrm{J}$ software was used to detected the band intensities. The relative protein level was normalized to that of GAPDH. The primary antibodies including anti-LC3A/B antibody (1:500, AF5402, Affinity, USA), anti-Beclin 1 antibody (1:500, AF5128, Affinity, USA), phospho-AMPK alpha (Thr172) antibody (1:500, AF3423, Affinity, USA), anti-AMPK alpha antibody (1:500, AF6423, Affinity, USA), phospho-mTOR (Ser2448) antibody (1:500, AF3308, Affinity, USA) and anti-mTOR antibody (1:500, AF6308, Affinity, USA), anti-GAPDH antibody (1:3000, AF7021, Affinity, USA).

\section{Effect of Dorsomorphin $2 \mathrm{HCl}$ on $\mathrm{C} 28 / \mathrm{I} 2$ cells}

The inhibitor of AMPK was used to assess the mechanism of polydatin on the C28/I2 cells. The C28/I2 cells were divided into four groups: control (only DMEM/F-12), LPS (DMEM/F-12 contain $5 \mu \mathrm{g} / \mathrm{mL}$ LPS, incubate for $24 \mathrm{~h}$ ), LPS+polydatin (DMEM/F-12 contain $5 \mu \mathrm{g} / \mathrm{mL}$ LPS and $1 \mu \mathrm{M}$ polydatin, incubate for 24 
h), and LPS+polydatin+Dorsomorphin $2 \mathrm{HCl}$ (After treated with $10 \mu \mathrm{M}$ Dorsomorphin $2 \mathrm{HCl}$ for $1 \mathrm{~h}, \mathrm{C} 28 / \mathrm{l} 2$ cells were cultured in DMEM/F-12 with $5 \mu \mathrm{g} / \mathrm{mL}$ LPS and $1 \mu \mathrm{M}$ polydatin for $24 \mathrm{~h}$ ) [15]. Then, the role of Dorsomorphin $2 \mathrm{HCl}$ on the proliferation and apoptosis of $\mathrm{C} 28 / \mathrm{I} 2$ cells and the mechanism were also detected according to the above description.

\section{Statistical analysis}

All the assay data were analyzed by SPSS 19.0 statistical software. The data were shown as ' $\chi \pm \mathrm{s}$. Multigroup comparison were analysed by One-way-ANOAY. Inter-group comparisons were analysed by SNK. Variance data were analysed by Kruskal-Wallis $\mathrm{H}$ test. $\mathrm{P}<0.05$ was seen as statistically significant.

\section{Result}

\section{In vivo}

\section{Polydatin attenuate pathological changes of cartilage tissues in KOA rats}

HE staining was performed to detected the pathological changes in of rats. Figure 1 reflected that the surface of knee cartilage tissues in control rats was smooth, the structure of cartilage was normal, chondrocytes were arranged orderly and no fracture in normal rats. However, compared with the control rats, the surface of cartilage was rough, chondrocytes were disordered, fracture, necrotic chondrocytes were observed in KOA rats $(P<0.01)$. Compared with the KOA rats, after treated with polydatin, there was less necrotic chondrocytes were observed, the arranged of chondrocytes and structure of cartilage was similar to control rats $(P<0.01)$.

\section{Polydatin inhibit apoptosis of chondrocytes in KOA rats}

TUNEL staining showed the apoptosis of chondrocytes in KOA rats (Figure 2). The TUNEL positive cell rate was significantly increased in KOA rats versus the normal rats $(P<0.01)$. And chondrocytes were reduced in volume, arranged loosely and chromatin condensed. However, polydatin reduced the TUNEL positive cell rate and ameliorated cell morphology significantly $(P<0.01)$. It implied that polydatin inhibited the chondrocytes apoptosis in joint tissues of KOA rats.

\section{Polydatin activated the autophagy of chondrocytes in KOA rats}

In order to detected the relation between polydatin and autophagy, Western blotting assay was performed to detected the level of autophagy-related proteins. As shown in Figure 3, the ratio of LC3II/LC3I was decreased in the KOA rats $(P<0.01)$, while Beclin-1 expression was markedly decreased compared with the control rats $(\mathrm{P}<0.05)$. On the other hand, polydatin reduced the LC3II/LC3I ratio and improved Beclin-1 expression $(P<0.01)$.

\section{In vitro}


To explore the effect of polydatin in osteoarthritis pathogenesis, MTT experiment was processed. The viability of $C 28 / 12$ cells induced by LPS was much lower than that of normal $C 28 / 12$ cells $(P<0.01)$, while polydatin evidently promoted the proliferation of $C 28 / 12$ cells induced by LPS $(P<0.01)$. It indicated that polydatin significantly ameliorated the proliferation of C28/I2 cells (Figure $4 A, B$ ).

\section{The effect of polydatin on the apoptosis of C28/I2 cells}

The C28//2 cells from the control, LPS and LPS+polydatin groups were estimate using flow cytometry. Compared with control C28/I2 cells, the proportion of apoptotic cells in the LPS induced C28/I2 cells was obviously increased $(P<0.01)$, while that was significantly reduced after treated with polydatin $(P<0.01)$ (Figure 4C,D).

\section{The effect of polydatin on the autophagy of C28/I2 cells}

We also detected the relation between polydatin and autophagy in vitro by Western blotting assay. Consistent with the results in vivo, compared with the normal C28/I2 cells group, the ratio of LC3II/LC3I was also decreased and the expression of Beclin- 1 was obviously decreased in the C28/I2 cells induced by LPS $(P<0.01 \& P<0.05)$. Compared with the $C 28 / 12$ cells induced by LPS, the LC3II/LC3I ratio was markedly improved and the expression of Beclin-1 was increased in $\mathrm{C} 28 / \mathrm{I} 2$ cells treated with polydatin $(P<0.01$, Figure $5 A \sim C)$.

\section{The effect of polydatin on the AMPK/mTOR signaling pathways}

mTOR acts a key role in molecule regulating cell autophagy. In order to detected the mechanism of the activation of polydatin on autophagy in C28/I2 cells, we detected the protein expression of mTOR and AMPK by Western blotting. As shown in the Figure 5D F, the expression of $p$-AMPK was reduced in LPS group compared with normal group $(P<0.05)$, while that of $p-m T O R$ was increased $(P<0.05)$. When LPS group cells treated with polydatin, the expression of $p$-AMPK was obviously increased and that of $p$ mTOR was obviously decreased respectively $(P<0.01)$. It implied that polydatin might regulate autophagy through the AMPK/mTOR signaling pathways.

\section{Dorsomorphin $2 \mathrm{HCl}$ blocked the improvement of polydatin on the LPS-induced $\mathrm{C} 28 / \mathrm{l} 2$ cells}

To further determine whether polydatin improved LPS-induced C28/I2 cells through AMPK/mTOR signaling pathways. The inhibitor of AMPK (Dorsomorphin $2 \mathrm{HCl}$ ) was used to block the activation of AMPK/mTOR signaling pathways. As shown in Figure 6, after co-treatment with polydatin and Dorsomorphin $2 \mathrm{HCl}$, the promotion of polydatin on the proliferation of $\mathrm{C} 28 / 12$ cells was reduced, while the inhibition of polydatin on the apoptosis of C28/I2 cells was blocked. Meanwhile, compared with the C28/I2 cells treated with polydatin, the LC3II/LC3I ratio and the expression of Beclin- 1 was markedly decreased in $\mathrm{C} 28 / \mathrm{I} 2$ cells $(\mathrm{P}<0.01$, Figure $7 \mathrm{~A} \sim \mathrm{C})$. It means that the activation of autophagy was significantly inhibited by Dorsomorphin $2 \mathrm{HCl}$. What's more, Western blotting results showed the expression of $\mathrm{p}-\mathrm{mTOR}$ was improved and that of $\mathrm{p}$-AMPK was decreased compared with LPS+polydatin 
group $(P<0.05, P<0.01)$ (Figure $7 D \sim F)$. It further confirmed that polydatin regulate autophagy through the AMPK/mTOR signaling pathways.

\section{Discussion}

KOA is the commonest chronic arthropathy characterized of lost cartilage, formation of osteophyte and sclerosis of subchondral bone, it is further resulting in disability, pain and affect the quality of life [16]. Polydatin can regulate the autophagy by AMPK/mTOR signaling pathway. The molecular mechanisms of polydatin in KOA rat models and C28/12 cells model was explored in this study. The results showed that polydatin activated autophagy and inhibited apoptosis by AMPK/mTOR signaling pathway.

Cartilage is consist of chondrocytes and is the major component of synovial joints. And chondrocytes have positive effect on the regeneration of hyaline cartilage [17]. In KOA, there are lots of physiological changes in the synovial membrane, cartilage, and bone structures, such as the rough surface of cartilage, disordered chondrocytes and fracture [18]. In this study, we found that polydatin can ameliorate the physiological changes in cartilage tissues of KOA rats according to the HE staining.

In our study, the apoptotic rate was both increased in the cartilage of KOA rats and LPS-induced C28/I2 cells. After treated with polydatin, the apoptotic rate was decreased both in vivo and in vitro. In addition, the proliferation of $\mathrm{C} 28 / \mathrm{I} 2$ cells were also significantly increased after treated with polydatin. For $\mathrm{OA}$ patients, the incidence of apoptosis in chondrocytes is much higher than normals [19], and the severity of apoptosis is related to the degree of articular cartilage destruction [20]. Netherlands did a large sample microarray study and found that the expression of apoptosis-related molecules in hematocyte of $O A$ patients were improved [21]. And the same thing, we also confirmed that the key effect of chondrocyte apoptosis in the progress of KOA, and polydatin reduced the apoptosis of chondrocyte and C28/I2 cells.

On the other hand, in KOA chondrocytes, a large number of alterations were observed in various homeostatic mechanisms, such as autophagy and mitochondrial function reduced, and reactive oxygen species (ROS) production increased [22]. Autophagy is a protective energy-conserving mechanism, and it transfers dysfunctional organelles or macromolecules into their constituent molecules during cell stress and starvation [23]. During autophagy, newly formed double-membraned vesicles envelope dysfunctional protein to make up autophagosomes, then it is fused with lysosomes and resulting in lysosomal breakdown [24]. Compared with non-OA control, the activity of autophagy pathways in the cartilage of OA patients was inhibited, and it means that autophagy pathways might related to the pathogenesis of OA cartilage [25]. Our results showed that the expression of autophagy-related proteins and autophagic activity in the articular cartilage of KOA rats and C28/I2 cells was lower than that in the normals. However, the activity of autophagic was activated by polydatin treatment. In OA cartilage and chondrocytes, the expression of autophagy-related proteins (Beclin1 and LC3II/I) was decreased, and the levels of the signaling factors related to apoptotic were improved [26]. Therefore, we hypothesized that the damaged chondrocytes could self-protection by inhibit the autophagy. 
Crucial molecules and pathways of AMPK/mTOR have been shown to regulate autophagy and received the most attention from researchers [27]. mTOR could improve the autophagy activity and reduce the apoptosis of chondrocytes in a KOA, further reduce the degree of OA [28]. It has been proved that polydatin regulated important molecules involved in autophagy and inflammation signaling pathways in bovine mammary epithelial cells [29], acute kidney injury [30,31] and myocardial injury in diabetic rats $[32,33]$. In this study, it was found that the effects of polydatin on autophagy induction was mTORdependent and regulated by AMPK. In addition, the activation of polydatin on autophagy was blocked by the inhibitor of AMPK (Dorsomorphin $2 \mathrm{HCl}$ ). These results revealed that the activation of chondrocyte autophagy induced by polydatin can inhibit apoptosis, and the mechanism might related to AMPK/mTOR signaling pathway.

In conclusion, by establishing a KOA model both in vivo and in vitro, we investigated the possible effect of polydatin for the treatment of KOA. The results demonstrated that polydatin treatment could ameliorate the pathological damage and reduce the chondrocyte apoptosis in cartilage tissues of KOA rats; increase the cell activity, inhibite apoptosis and activate autophagy of LPS-stimulated C28/I2 cells. At the same time, we found that polydatin displayed the pro-autophagic activity in KOA via the AMPK/mTOR pathway. These findings suggested polydatin as a potential therapeutic agent for KOA.

\section{Abbreviations}

\begin{tabular}{|ll|}
\hline KOA & Knee osteoarthritis \\
\hline SD & Sprague-Dawley \\
\hline HE & Hematoxylin-eosin \\
\hline TUNEL & Terminal deoxynucleotidyl transferase dUTP nick end labeling \\
\hline RIPA & Radioimmunoprecipitation assay \\
\hline PVDF & polyvinylidene difluoride \\
\hline ECL & Enhanced chemiluminescence \\
\hline ROS & reactive oxygen species \\
\hline
\end{tabular}

\section{Declarations}

\section{Acknowledgments}

Not applicable.

\section{Authors' contributions}

Zhengcong Ye and Chun He designed the research and acquisition of data, Pengzheng Yu and Guoping Cao analyzed the data. Canfeng Wang drafted the manuscript. Qinrong Shen revised the manuscript for 
important intellectual content.

\section{Data availability statement}

All data generated or analyzed during this study are included in this article.

\section{Funding statement}

The work was supported by the Traditional Chinese Medicine Science and Technology Project of Zhejiang Province (No. 2019ZA107).

\section{Ethics approval and consent to participate}

The animal experiments were in accordance with the guidelines of laboratory animal care and were approved by the Hangzhou Eyong Biotechnological Co., Ltd. Animal Experiment Center (Hangzhou, China).

\section{Consent for publication}

Not applicable.

\section{Competing interests}

The authors declare that there are no conflicts of interest.

\section{References}

1. He B., \& Jiang D. (2019). Hotair-induced apoptosis is mediated by sponging mir-130a-3p to repress chondrocyte autophagy in knee osteoarthritis. Cell Biology International, 44(2).

2. Wick M.C., Kastlunger M., Weiss R.J. (2014). Clinical imaging assessments of knee osteoarthritis in the elderly: a mini-review. Gerontology, 60: 386-94.

3. Kerkhof H.J., et al. (2014). Prediction model for knee osteoarthritis incidence, including clinical, genetic and biochemical risk factors. Ann Rheum Dis, 73(12): 2116-21.

4. Dulay G.S., Cooper C., Dennison E.M. (2015). Knee pain, knee injury, knee osteoarthritis \& work. Best practice \& research Clinical rheumatology, 29: 454-61.

5. Funato S., Yasuhara R., Yoshimura K., Miyamoto Y., Kaneko K., Suzawa T., Chikazu D., Mishima K., Baba K., Kamijo R. (2017). Extracellular matrix loss in chondrocytes after exposure to interleukin-1 $\beta$ in NADPH oxidase-dependent manner. Cell Tissue Res. 368: 135-144.

6. Hosseinzadeh A., Kamrava S.K., Joghataei M.T., Darabi R., Shakeri-Zadeh A., Shahriari M., Reiter R.J., Ghaznavi H., Mehrzadi S. (2016). Apoptosis signaling pathways in osteoarthritis and possible protective role of melatonin. J Pineal Res., 61: 411-425. 
7. Caramés B., Hasegawa A., Taniguchi N., Miyaki S., Blanco F.J., Lotz M. (2012). Autophagy activation by rapamycin reduces severity of experimental osteoarthritis. Ann Rheum Dis., 71: 575-581.

8. Şöhretoğlu D., Baran M.Y., Arroo R., Kuruüzüm-Uz A. (2018). Recent advances in chemistry, therapeutic properties and sources of polydatin. Phytochem. Rev., 17: 973-1005.

9. Ye J., Piao H., Jiang J., Jin G., Zheng M., Yang J., Jin X., Sun T., Choi Y.H., Li L., et al. Polydatin inhibits mast cell-mediated allergic inflammation by targeting PI3K/Akt, MAPK, NF-kappaB and Nrf2/HO-1 pathways. (2017). Sci. Rep., 7: 11895.

10. Zakhary A.S., Aboul-Gheit A.K., \& Ghoneim S.A. (2014). Fuel rich and fuel lean catalytic combustion of the stabilized confined turbulent gaseous diffusion flames over noble metal disc burners. Egyptian Journal of Petroleum, 23(1).

11. Gu T.T., Zhang D.M., Wan Z.Y., Li T.S., \& Kong L.D. (2020). Polydatin enhances glomerular podocyte autophagy homeostasis by improving nrf2-dependent antioxidant capacity in fructose-fed rats. Molecular and Cellular Endocrinology, 520: 111079.

12. Liao H, Zhang Z, Liu Z, Lin W, Huang J., \& Huang Y. (2020). Inhibited microrna-218-5p attenuates synovial inflammation and cartilage injury in rats with knee osteoarthritis by promoting sclerostin sciencedirect. Life sciences, 267: 118893.

13. Song X.Y., Xie W.P., Zhang P., Zhao M., \& Bi R.X. (2020). Cangxitongbi capsule protects articular cartilage of the knee in rats by regulating adamts-5. Annals of Translational Medicine, 8(22): 15111511.

14. Wongwat T., Srihaphon K., Pitaksutheepong C., Pitaksuteepong T. (2020). Suppression of inflammatory mediators and matrix metalloproteinase (MMP)- 13 by Morus alba stem extract and oxyresveratrol in RAW 264.7 cells and C28/I2 human chondrocytes. J Tradit Complement Med., 10(2): 132-140.

15. Li Y., Liu M., Song X., Zheng X., \& Yang, J. (2020). Exogenous hydrogen sulfide ameliorates diabetic myocardial fibrosis by inhibiting cell aging through sirt6/ampk autophagy. Frontiers in Pharmacology, 11: 1150.

16. Ma Z, Huang Z, Zhang L, Li X, \& Wang P. (2021). Vanillic acid reduces pain-related behavior in knee osteoarthritis rats through the inhibition of nlrp3 inflammasome-related synovitis. Frontiers in Pharmacology, 11: 599022.

17. Zhang Q., Lai S., Hou X., Cao W., Zhang Y., Zhang Z. (2018). Protective effects of PI3K/Akt signal pathway induced cell autophagy in rat knee joint cartilage injury. Am J Transl Res., 10: 762-770.

18. Wu Z., Luan Z., Zhang X., Zou K., Ma S., Yang Z., Feng W., He M., Jiang L., Li J., Yao J. (2019). Chondro-protective effects of polydatin in osteoarthritis through its effect on restoring dysregulated autophagy via modulating MAPK, and PI3K/Akt signaling pathways. Sci Rep., 9: 13906.

19. Héraud F., Héraud A., Harmand M.F. (2000). Apoptosis in normal and osteoarthritic human articular cartilage. Ann Rheum Dis., 59: 959-965.

20. Thomas C.M., Fuller C.J., Whittles C.E., Sharif M. (2011). Chondrocyte death by apoptosis is associated with the initiation and severity of articular cartilage degradation. Int J Rheum Dis., 14: 
191-198.

21. Ramos Y.F., Bos S.D., Lakenberg N., Böhringer S., den Hollander W.J., Kloppenburg M., Slagboom P.E., Meulenbelt I. (2014). Genes expressed in blood link osteoarthritis with apoptotic pathways. Ann Rheum Dis., 73: 1844-1853.

22. Friedman B., Corciulo C., Castro C.M., \& Cronstein B.N. (2021). Adenosine a2a receptor signaling promotes foxo associated autophagy in chondrocytes. Scientific Reports.,11(1): 968.

23. Carames B., Taniguchi N., Otsuki S., Blanco F.J., Lotz M. (2010). Autophagy is a protective mechanism in normal cartilage, and its aging-related loss is linked with cell death and osteoarthritis. Arthritis. Rheum., 62: 791-801.

24. Choi A.M., Ryter S.W., Levine B. (2013). Autophagy in human health and disease. N. Engl. J. Med., 368: 1845-1846.

25. Wang F., Liu J., Chen X., Zheng X., Qu N., Zhang B., Xia C. (2019). IL-1ß receptor antagonist (IL-1Ra) combined with autophagy inducer (TAT-Beclin1) is an effective alternative for attenuating extracellular matrix degradation in rat and human osteoarthritis chondrocytes. Arthritis Res Ther., 21: 171.

26. Caramés B., Taniguchi N., Otsuki S., Blanco F.J., Lotz M. (2010). Autophagy is a protective mechanism in normal cartilage, and its aging-related loss is linked with cell death and osteoarthritis. Arthritis Rheum., 62: 791-801.

27. Xiong Y., Yepuri G., Forbiteh M., Yu Y., Montani J.P., Yang Z., Ming X.F. (2014). ARG2 impairs endothelial autophagy through regulation of MTOR and PRKAA/AMPK signaling in advanced atherosclerosis. Autophagy, 10: 2223-2238.

28. Shen J.M., Feng L., Chen J., Wu Y., Yu J. (2017). Effect of Simiaosan on apoptosis and autophagy of chondrocyte in treating knee osteoarthritis in rats. New Chin Med., 49: 12-15.

29. Fu Y.R., Jin Y.C., Shan A.S., Zhang J., Tang H.Y., \& Shen J.L., et al. (2021). Polydatin protects bovine mammary epithelial cells against zearalenone-induced apoptosis by inhibiting oxidative responses and endoplasmic reticulum stress. Toxins, 13(2): 121.

30. Deng Z., Sun M., Wu J., Fang H., Cai S., \& An S., et al. (2021). Sirt1 attenuates sepsis-induced acute kidney injury via beclin1 deacetylation-mediated autophagy activation. Cell Death \& Disease, 12(2): 217.

31. El-Hameed A. (2020). Polydatin-loaded chitosan nanoparticles ameliorates early diabetic nephropathy by attenuating oxidative stress and inflammatory responses in streptozotocin-induced diabetic rat. Journal of Diabetes \& Metabolic Disorders, 19(2): 1599-1607.

32. Tan Y.Y., Chen L.X., Fang L., \& Zhang Q. (2020). Cardioprotective effects of polydatin against myocardial injury in diabetic rats via inhibition of nadph oxidase and $\mathrm{nf}-\mathrm{kb}$ activities. BMC Complementary Medicine and Therapies, 20(1).

33. Mostafa F., Abdel-Moneim A., Abdul-Hamid M., Galaly S.R., \& Mohamed H.M.. (2021). Polydatin and polydatin-loaded chitosan nanoparticles attenuate diabetic cardiomyopathy in rats. Journal of Molecular Histology, 52(2): 135-152. 
Figures
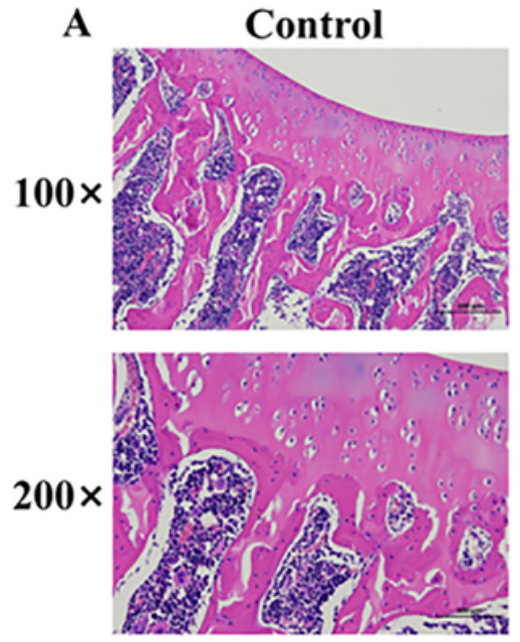

KOA
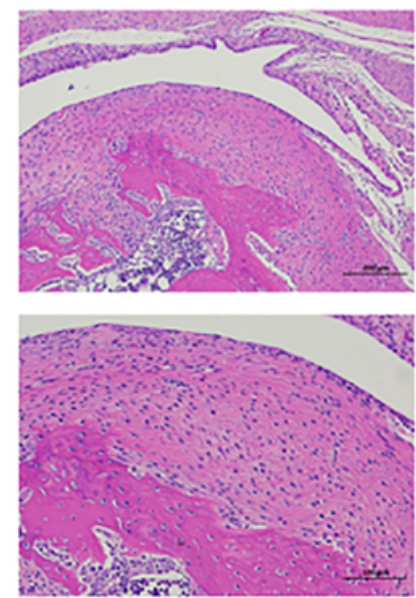

KOA+polydatin
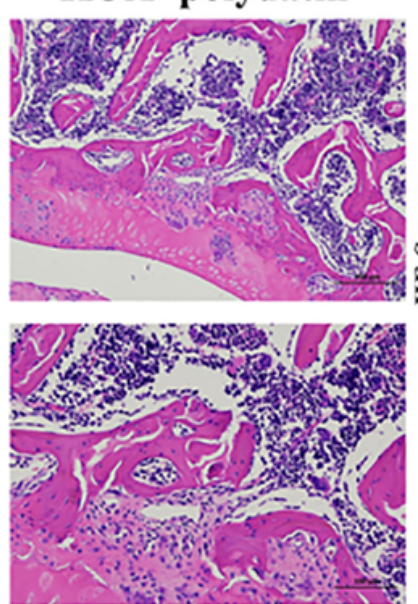

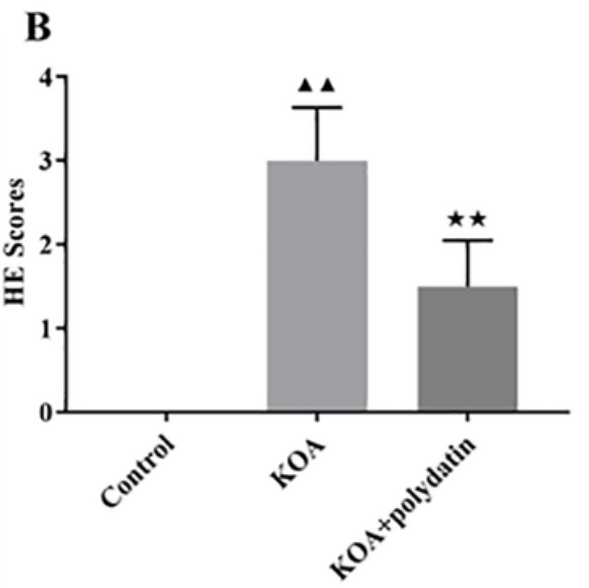

Figure 1

Polydatin ameliorate the pathological changes of cartilage tissues in KOA rats. A, HE staining images in cartilage tissues of each group rats (original magnification 100x, 200x). B, Semi-quantitative analysis of the histological lesions. $(\bar{x} \pm s, n=10), \Delta P<0.05, \Delta \Delta P<0.01$ vs. control, $\otimes P<0.05, \triangle \otimes P<0.01$ vs. KOA.
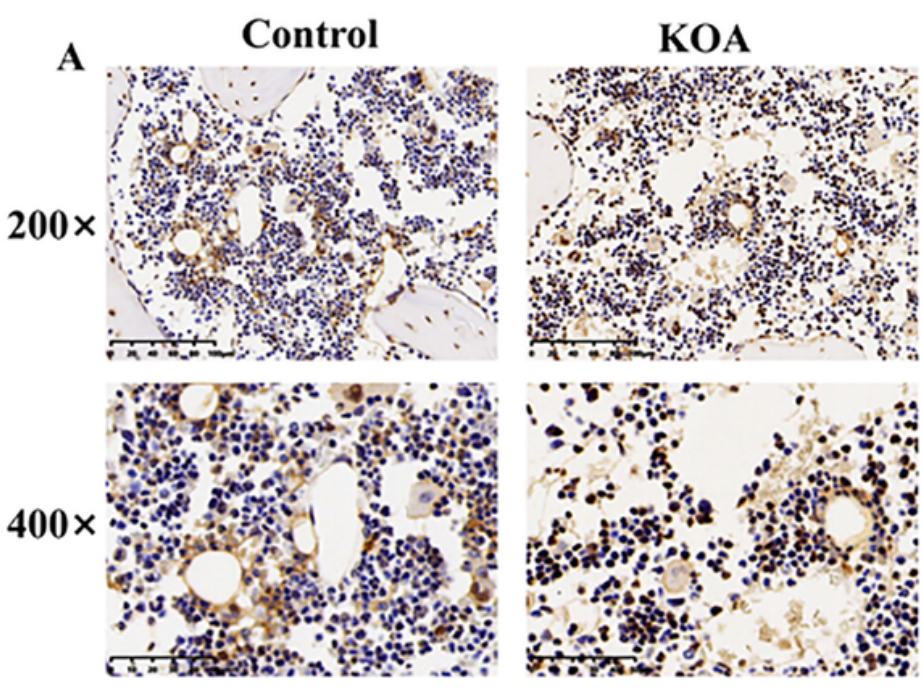

KOA+polydatin

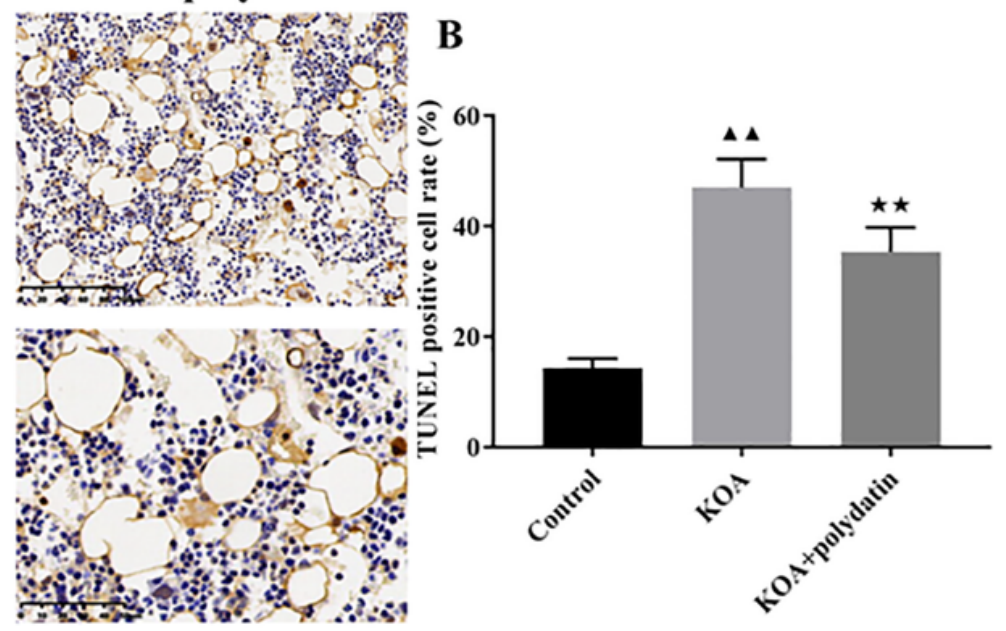

Figure 2

Polydatin inhibit the apoptosis of chondrocytes in joint tissues of KOA rats. A, TUNEL staining images in rats' joint tissues (original magnification 200x, 400x); B, Semi-quantitative assessment of TUNEL positive cell rate in rats' joint tissues. $(\bar{x} \pm s, n=10), \Delta P<0.05, \Delta \Delta P<0.01$ vs. control, $\triangle P<0.05, \mathbb{\Delta} \mathbb{\Delta}<<0.01$ vs. KOA. 


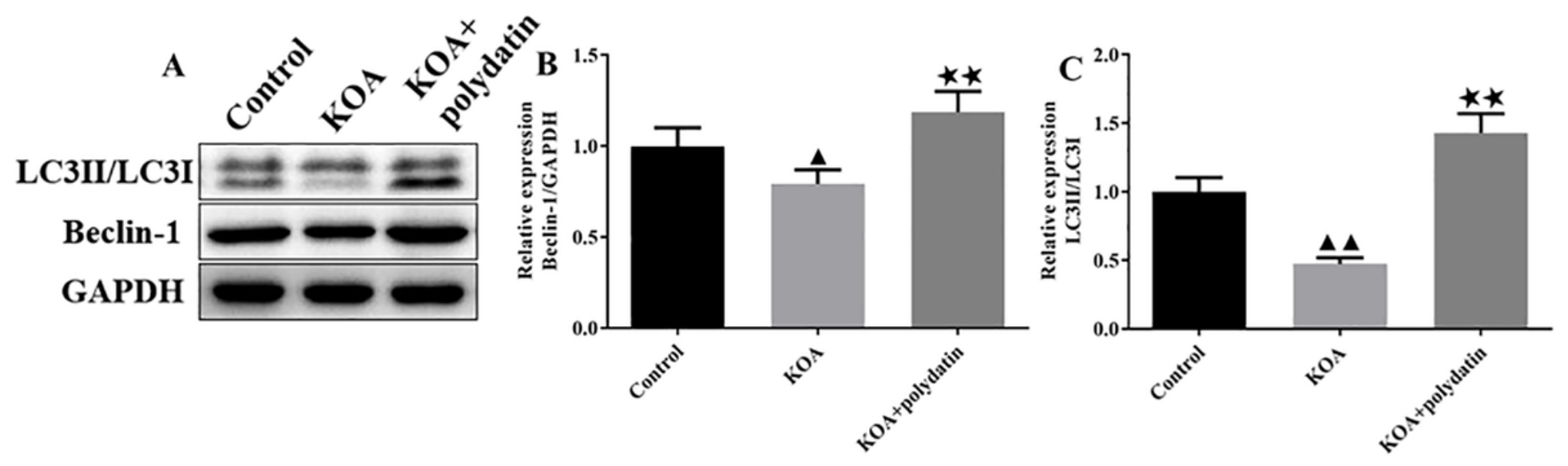

Figure 3

Expression of autophagy-related proteins LC3 and Beclin-1 in the cartilage tissue after treated with polydatin. A, Representative immunoblots of LC3 and Beclin-1 proteins in different treatment groups. $B$ and C, Quantitative densitometric analysis of LC3 and Beclin-1. ( $\bar{x} \pm s, n=3), \Delta P<0.05, \Delta \Delta P<0.01$ vs. control, $\otimes P<0.05, \mathbb{Q} \mathrm{P}<0.01$ vs. KOA.
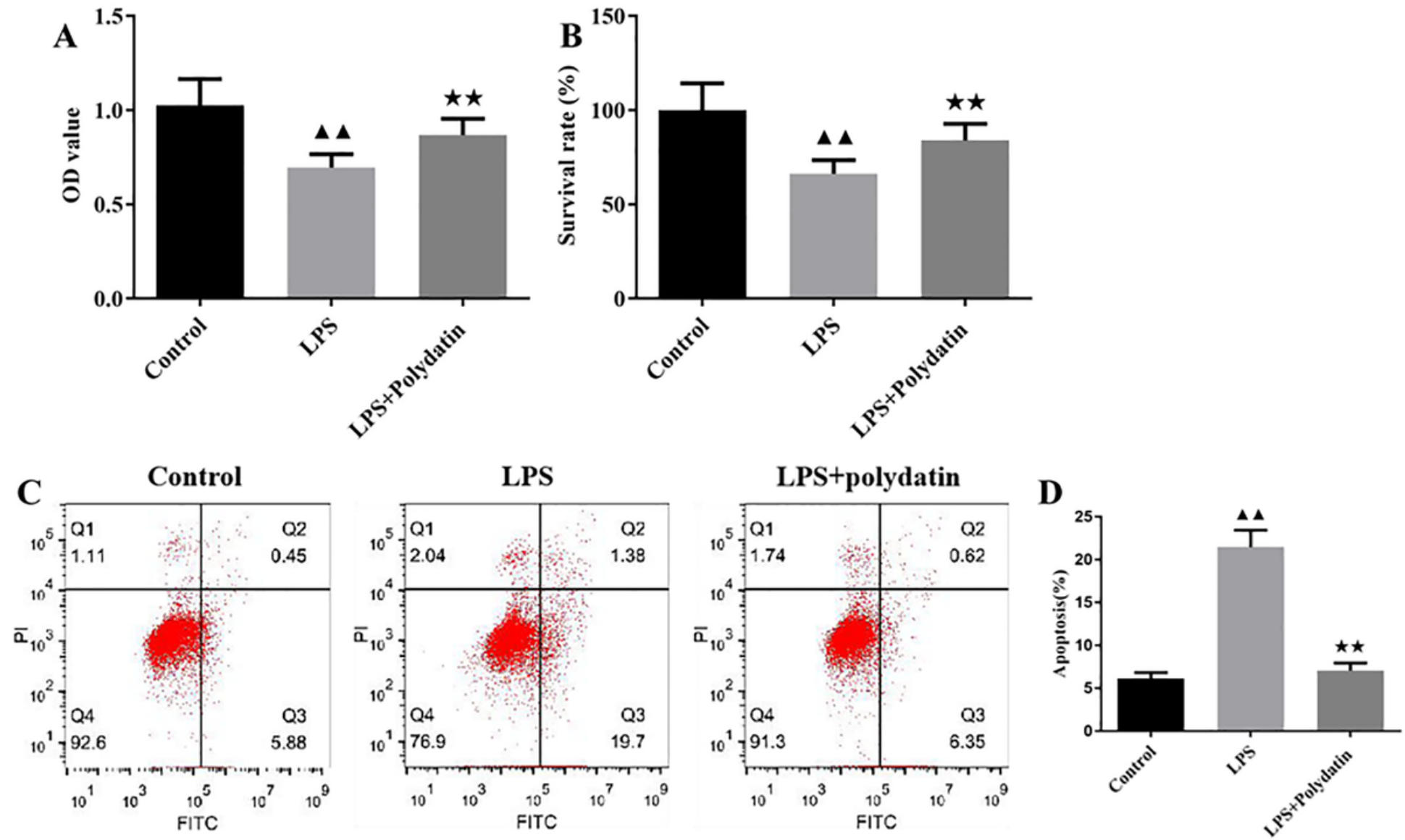

Figure 4

The viability and apoptotic of $C 28 / \mathrm{I} 2$ cells was detected by MTT assays and flow cytometry. $A$ and $B$, the OD value and survival rate in control, LPS and LPS+polydatin group. C and D, flow cytometry detected the 
apoptotic of C28//2 cells in control, LPS and LPS+polydatin group. ( $\bar{x} \pm s, n=3), \Delta P<0.05, \Delta \Delta P<0.01$ vs.

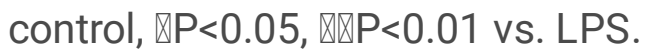

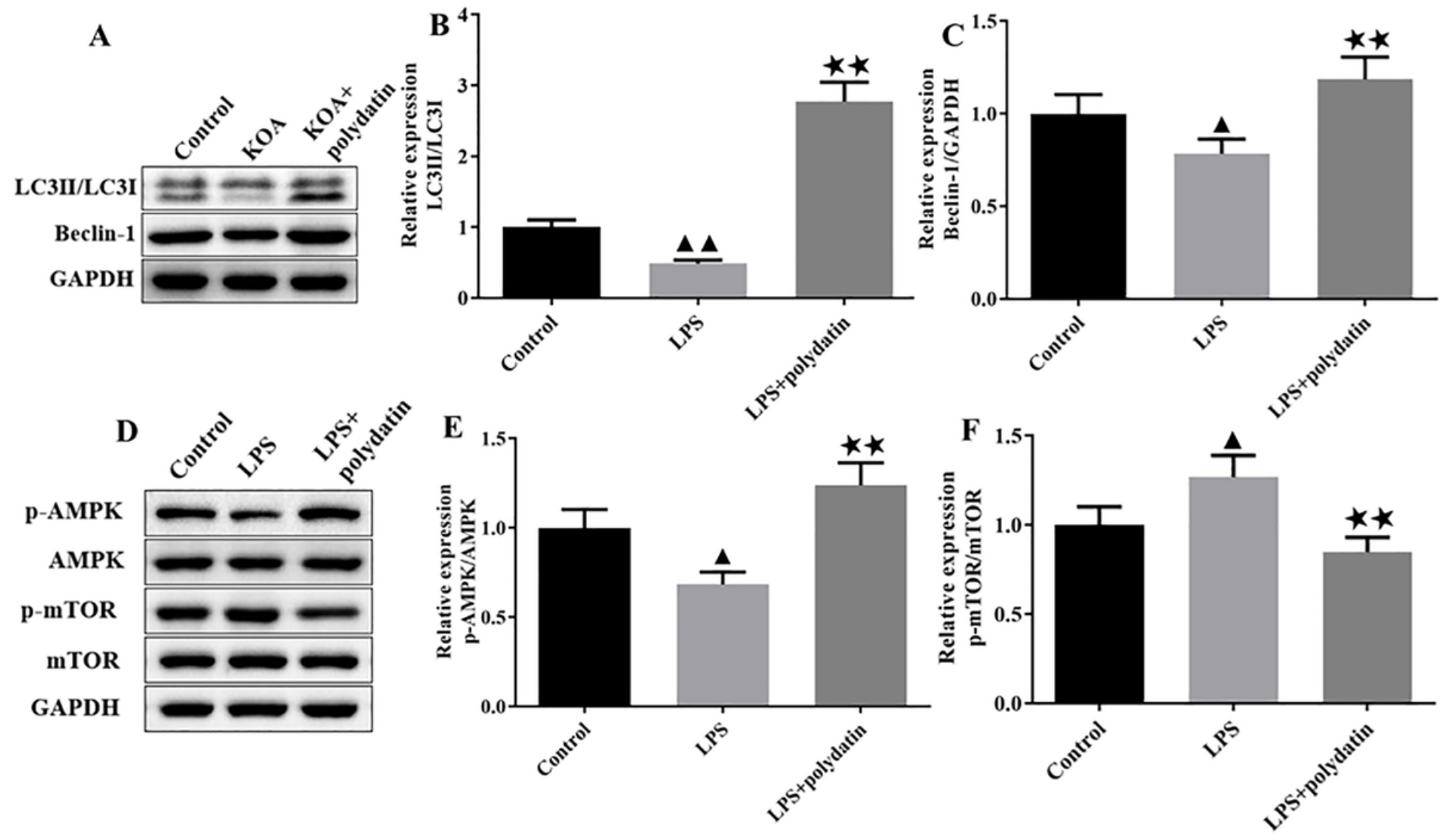

Figure 5

Polydatin ameliorated articular cartilage degeneration might occur through autophagy regulation mediated by the AMPK/mTOR signaling pathway. $A$ and $D$, Representative immunoblots of each protein in different treatment groups. B, C, E and F, Quantitative densitometric analysis of LC3, Beclin-1, p-AMPK, AMPK, $p-m T O R$ and mTOR. ( $\bar{x} \pm s, n=3), \Delta P<0.05, \Delta \Delta P<0.01$ vs. control, $\triangle P<0.05$, $\mathbb{\Delta} P<<0.01$ vs. LPS. 

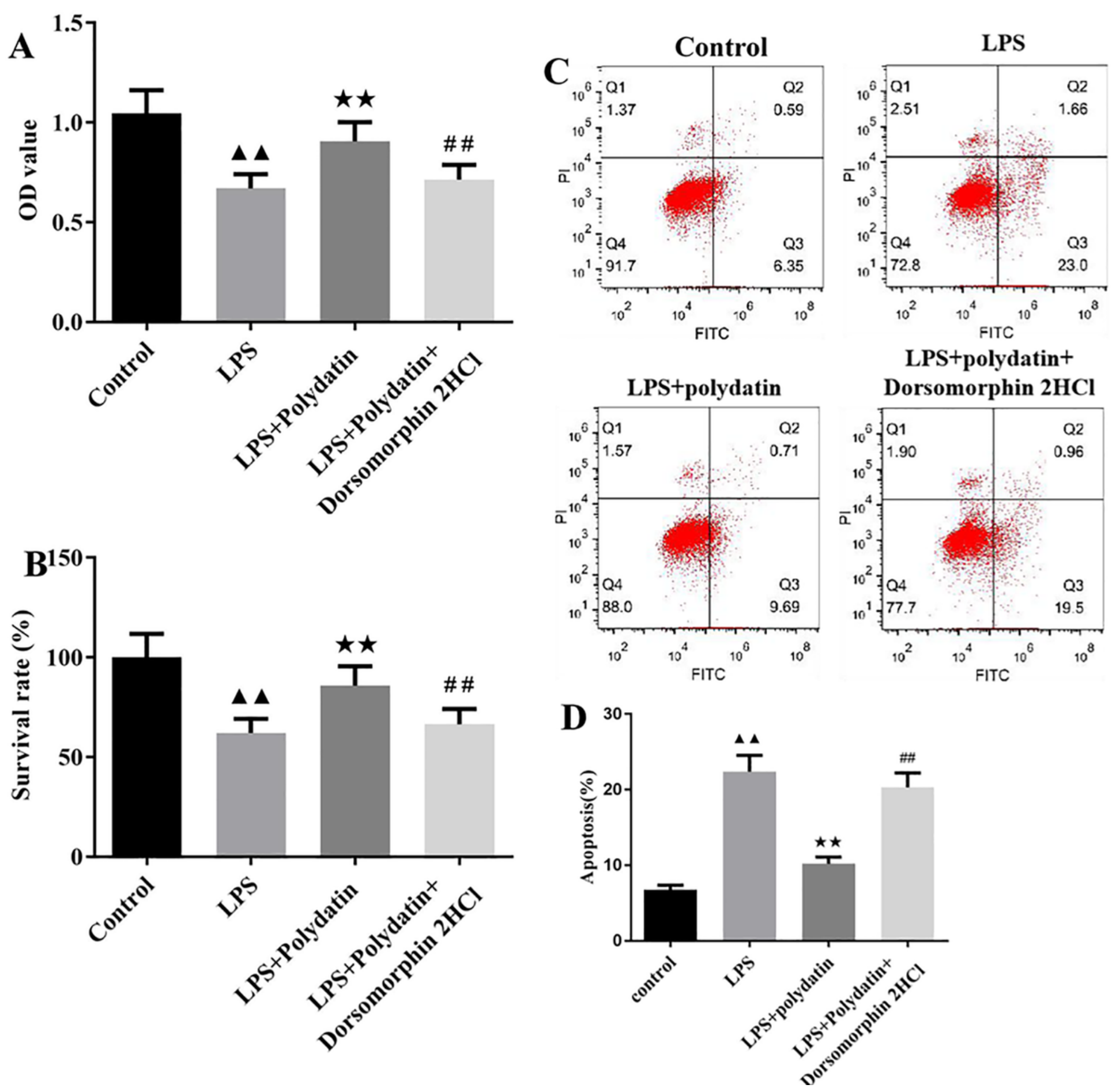

Figure 6

The effect of polydatin and Dorsomorphin $2 \mathrm{HCl}$ on the viability and apoptotic analysis of $\mathrm{C} 28 / \mathrm{I} 2 \mathrm{cells}$. A and $B$, the OD value and survival rate in control, LPS, LPS+polydatin and LPS+polydatin+Dorsomorphin $2 \mathrm{HCl}$ group. $\mathrm{C}$ and $\mathrm{D}$, flow cytometry detected the apoptotic of $\mathrm{C} 28 / \mathrm{l} 2$ cells in control, LPS, LPS+polydatin group and LPS+polydatin+Dorsomorphin $2 \mathrm{HCl}$ group. ( $\overline{\mathrm{x}} \pm \mathrm{s}, \mathrm{n}=3), \mathbf{\Delta}<<0.05, \mathbf{\Delta} \mathbf{\Delta} \mathrm{P}<0.01$ vs. control, $\triangle P<0.05, \mathbb{Q} \mathbb{P}<0.01$ vs. LPS, $\# \mathrm{P}<0.05, \# \# \mathrm{P}<0.01$ vs. LPS+polydatin. 

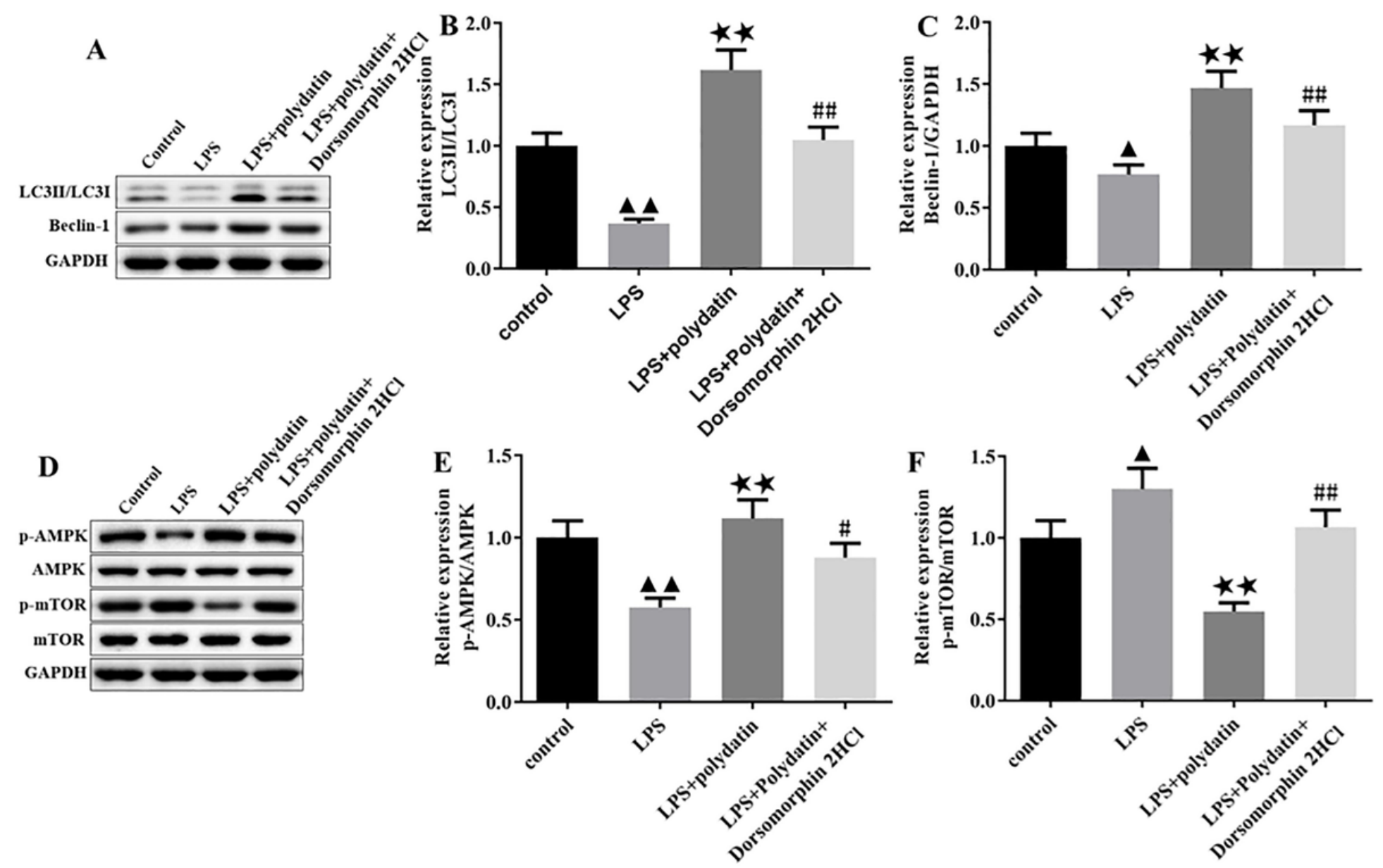

Figure 7

The improvement of polydatin on articular cartilage degeneration through autophagy regulation mediated by the AMPK/mTOR signaling pathway was blocked by Dorsomorphin $2 \mathrm{HCl}$. A, D. Representative immunoblots of each protein in different treatment groups. B, C, E and F, After different treatment, the expression of LC3, Beclin-1, p-AMPK, AMPK, p-mTOR and mTOR was verify by western blot in each group.

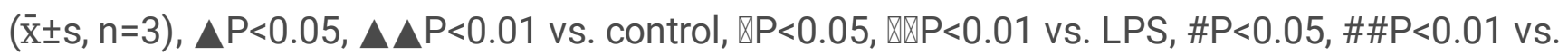
LPS+polydatin. 